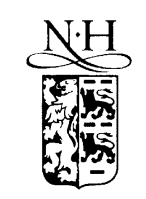

ELSEVIER

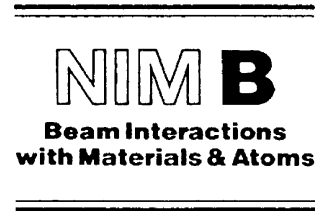

www.elsevier.com/locate/nimb

\title{
Improving neutron detection efficiency by using passive converters
}

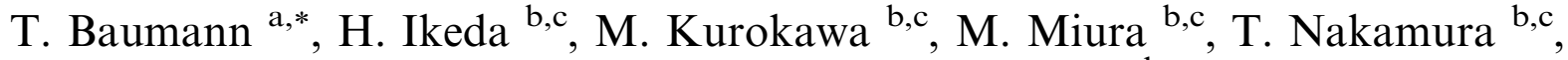 \\ Y. Nishi ${ }^{c}$, S. Nishimura ${ }^{c}$, A. Ozawa ${ }^{c}$, T. Sugimoto ${ }^{\text {b,c }}$, I. Tanihata ${ }^{c}$, \\ M. Thoennessen ${ }^{\mathrm{a}, \mathrm{d}}$ \\ ${ }^{a}$ National Superconducting Cyclotron Laboratory, Michigan State University, East Lansing, MI 48824-1321, USA \\ ${ }^{\mathrm{b}}$ Tokyo Institute of Technology, 2-12-1 O-Okayama, Meguro, Tokyo 152-8551, Japan \\ ${ }^{\mathrm{c}}$ RIKEN, 2-1, Hirosawa, Wako, Saitama 351-0198, Japan \\ d Department of Physics and Astronomy, Michigan State University, East Lansing, MI 48824, USA
}

Received 2 October 2001; received in revised form 16 November 2001

\begin{abstract}
The effect of passive iron converters on the detection efficiency of neutrons in a plastic scintillator was investigated at energies of 20-140 MeV. An enhanced detection efficiency for neutron energies above $70 \mathrm{MeV}$ was found using iron converters of 2-3 cm thickness. The experimental results are compared to a simulation. (C) 2002 Elsevier Science B.V. All rights reserved.
\end{abstract}

PACS: $29.40 . \mathrm{Mc}$

Keywords: Neutron detection; Intermediate energy; Passive converter

\section{Introduction}

The study of nuclei close to the neutron dripline and the investigation of nuclear systems even beyond the drip-line has greatly expanded in recent years. This increased interest is based on new phenomena, for example multiple neutron halos and the disappearance of shells at the neutron drip-line.

Rare isotope beam facilities that use projectile fragmentation and in-flight separation to produce

\footnotetext{
${ }^{*}$ Corresponding author. Tel.: +1-517-333-6437; fax: +1-517353-5967.

E-mail address: baumann@nscl.msu.edu (T. Baumann).
}

these neutron-rich nuclei require neutron detection systems with a high detection efficiency at energies of about $50-400 \mathrm{MeV}$ for their investigation. In many experimental studies, the neutrons are breakup products from the projectile which are detected in the forward direction, and the time of flight is used to determine the neutron energy. This technique generally requires large-area detectors with good time resolution and high detection efficiencies. The detection efficiency is especially important if multiple neutrons are to be measured in coincidence. Although liquid or plastic scintillators are usually well suited for large-area detectors, the interaction length of neutrons in these materials requires a rather deep detector volume in order to 
achieve high detection efficiencies. A possibility to boost neutron detection efficiency, particularly for higher neutron energies, is to use passive converter materials that have a much shorter interaction length for neutrons.

In this paper, we determine the lower energy limit for increasing the detection efficiency of a plastic scintillator by adding a passive iron converter. Passive converters are widely applied in neutron calorimeters used for high energy physics experiments. The large area neutron detector (LAND) at GSI also makes use of passive iron converters [1]. This detector is a time-of-flight wall optimized for neutrons with energies of up to 1 $\mathrm{GeV}$. While the LAND group reported detection efficiencies for their detector setup at energies of $200 \mathrm{MeV}$ and above, experimental data for an iron-plastic combination at energies around 100 $\mathrm{MeV}$ do not exist.

Here we present measurements of relative detection efficiencies for a combination of iron converter and plastic scintillator compared to a pure plastic scintillator detector for neutrons of 20 $140 \mathrm{MeV}$. We also show results from Monte Carlo simulations that complement the experimental data.

\section{Experimental technique}

The experiment was performed at the RIKEN Accelerator Research Facility. Elements of the NEUT neutron detector array (see e.g. Ref. [2]) were used for this measurement.

Neutrons of a broad energy range were produced using a $100 \mathrm{MeV} /$ nucleon beam of ${ }^{13} \mathrm{C}$ from the RIKEN Ring Cyclotron impinging on a $2 \mathrm{~cm}$ thick aluminum target. The measured energy distribution of the detected neutrons is displayed in Fig. 1. The primary beam was stopped in the target, so that only lighter fragments and neutrons could reach the detector setup. A thin plastic start detector was placed $10 \mathrm{~cm}$ in front of the production target, while the neutron detectors were mounted at a distance of about $5 \mathrm{~m}$ behind the target. This distance was needed in order to yield a sufficient energy resolution by a time-of-flight measurement. With a timing resolution of approx-

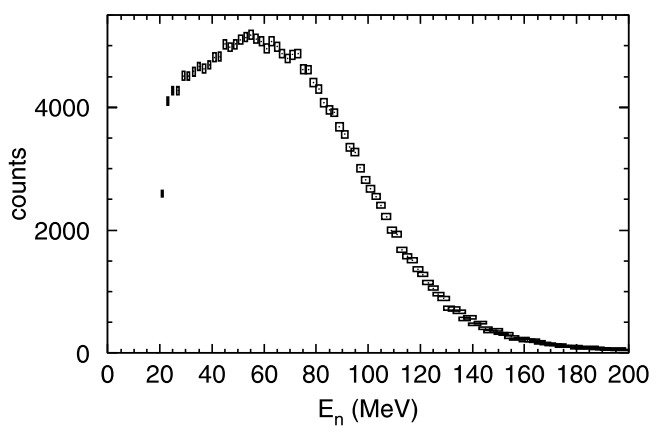

Fig. 1. Measured neutron energy distribution. The rectangles indicate the error based on the time-of-flight measurement and the statistical error.

imately $\sigma_{t}=170 \mathrm{ps}$, an energy resolution of $1 \%$ (in sigma) at $100 \mathrm{MeV}$ neutron energy was achieved.

The neutron detector setup (see Fig. 2) consisted of two sets which were placed symmetrically with respect to the beam axis. This assured the same neutron flux through both detector sets. Each set contained three blocks of $6 \times 6 \times 108 \mathrm{~cm}^{3}$ BC-408 plastic scintillators stacked vertically. Each scintillator block was read out by two photomultiplier tubes mounted on either end. The front of each set was covered by plastic veto detectors of $5 \mathrm{~mm}$ thickness in order to discriminate charged reaction products. The two detector sets were mounted roughly $40 \mathrm{~cm}$ above and below the beam axis.

Iron converters of 2 and $3 \mathrm{~cm}$ thickness were added to one of the two detector sets, enabling a direct comparison of the number of detected neutrons in each of the two detector sets. The iron converter was placed between the veto detectors and the neutron detectors. In order to account for the asymmetries of the setup, such as the fact that the detectors themselves might have detection efficiency differences, or small deviations from a symmetric geometry of the setup, a measurement without any iron converter was also performed. Data from this measurement was used in the analysis for the normalization of the two detector sets.

Separation of beam-related gamma rays and neutrons was achieved by the time-of-flight measurement. A small background of cosmic rays could not be discriminated because the BC-408 


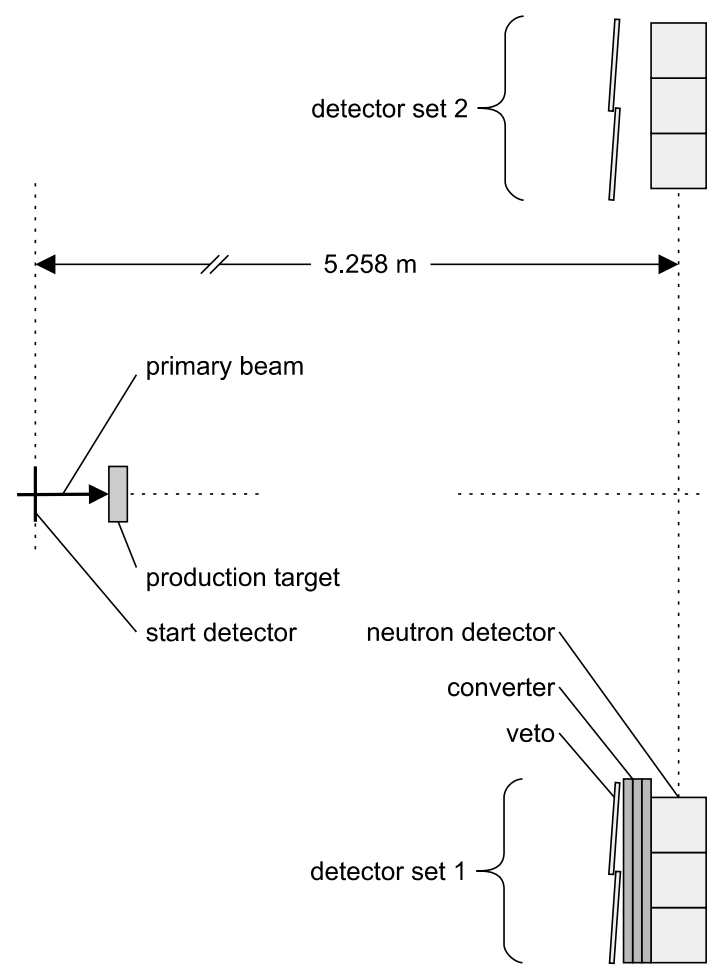

Fig. 2. Schematic cross-section of the experimental setup.

plastic scintillator does not offer pulse shape discrimination capabilities. It was possible to subtract an extrapolated background in the analysis, although the final results of the analysis showed that the amount of background events was negligible.

\section{Results}

The results of this measurement are presented as detection efficiency ratios in dependence of neutron energy. Since this investigation aimed at the question of the effectiveness of passive converters, a relative measurement was sufficient. The absolute detection efficiencies of plastic scintillator materials have been measured many times and can be calculated with good accuracy $[3,4]$.

The efficiency ratios were calculated by dividing the number of neutrons per energy bin that were detected with the converter-equipped detector set by the number of neutrons that the pure plastic scintillator detected. In the same way we deduced efficiency ratios from the measurement without iron converter-we will call these correction ratios. The correction ratios were used to account for the asymmetries of the setup and the detectors. We divided the efficiency ratios by the correction ratios in order to yield the corrected efficiency ratios.

If $\varepsilon_{1}$ corresponds to the absolute detection efficiency with iron converter, and $\varepsilon_{2}$ to the absolute efficiency of the plastic scintillator alone, our measurement provides the efficiency ratio $\varepsilon_{1} / \varepsilon_{2}$. Accordingly, an efficiency ratio above 1.0 would indicate an enhancement of the detection efficiency by the passive converter.

Position information was obtained by measuring the time difference of the signals from the left and right photo-multipliers. This enabled us to eliminate events at the far left and right end of the scintillator blocks, where the detectors were not covered with veto counters and iron converter.

Fig. 3(a) shows the results for a $2 \mathrm{~cm}$ converter (open circles) and a $3 \mathrm{~cm}$ converter (filled circles), both with a $2 \mathrm{MeVee}$ (electron equivalent, i.e. energy deposition equivalent to a $2 \mathrm{MeV}$ electron) threshold cut. The threshold cut was applied to the
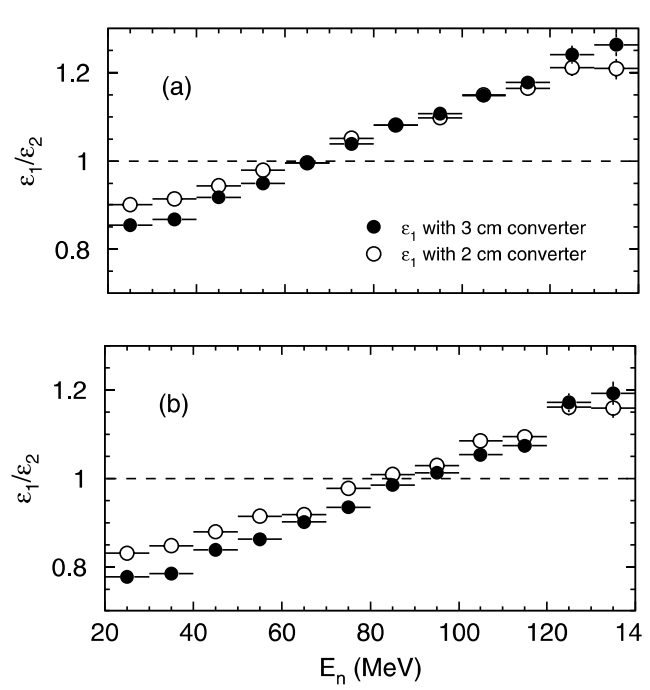

Fig. 3. Experimental efficiency ratios for the two different converter thicknesses. Open symbols correspond to the $2 \mathrm{~cm}$, filled symbols to the $3 \mathrm{~cm}$ converter. The upper panel (a) displays the ratios obtained with a $2 \mathrm{MeVee}$ threshold cut, while the lower panel (b) shows data from the same measurements, but with a $5 \mathrm{MeVee}$ threshold cut. 
mean signal amplitude of the left and the right photo-multiplier, with small geometric corrections taken into account. The plot of efficiency ratio versus neutron energy clearly shows the increase in detection efficiency of the iron-plastic combination as compared to the pure plastic detector with increasing neutron energy. At energies below about $70 \mathrm{MeV}$, the iron converter reduces the detection efficiency, but for neutrons of above 70 $\mathrm{MeV}$, the detection efficiency is enhanced. This is true for the $2 \mathrm{~cm}$ converter as well as for the $3 \mathrm{~cm}$ converter, with the efficiency curve rising more steeply in the latter case. The increase in detection efficiency is significant: at $100 \mathrm{MeV}$ it is about $10 \%$, at $140 \mathrm{MeV}$ even more than $20 \%$.

Data from the same measurements were also analyzed applying a $5 \mathrm{MeVee}$ threshold cut and the results for the two converter thicknesses are plotted in Fig. 3(b). The higher threshold setting shifts the efficiency ratios down, so that the crossover moves to a higher energy of about $90 \mathrm{MeV}$. The overall smaller efficiency ratios are due to the fact that the passive converter enhances the number of events with small signal amplitudes, therefore the effectiveness is directly dependent on the threshold setting. This is displayed in Fig. 4, which shows the measured energy spectrum of a detector set without converter (thick line) and one with a 3 $\mathrm{cm}$ iron converter (thin line). These signal amplitude spectra were obtained with a gate on the 90 $100 \mathrm{MeV}$ neutron energy range, so they reflect the

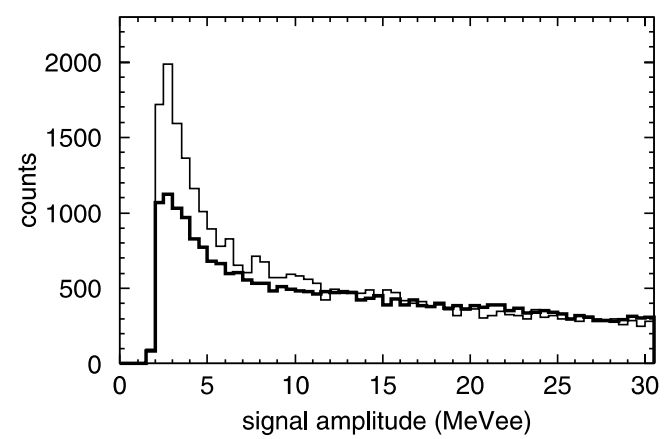

Fig. 4. Measured signal amplitudes for 90-100 MeV neutrons with a $2 \mathrm{MeVee}$ threshold cut. The thick-lined histogram represents the energy spectrum of a detector set without converter, while the thin-lined histogram shows the spectrum of a detector set with a $3 \mathrm{~cm}$ iron converter. signal amplitude in a range where the converter enhances detection efficiency. From these spectra, to which a 2 MeVee threshold cut was applied, it becomes clear that a higher threshold cut decreases the effectiveness of the passive converter.

\section{Simulation}

One of the objectives of this measurement was to corroborate results from Monte Carlo simulations of neutron detection efficiencies for cases in which passive converter materials were employed. We used the detector development tool GEANT in combination with the FLUKA and MICAP ${ }^{1}$ packages [5-8] extensively for the design of a new large-area high-efficiency neutron detector to be used at the coupled cyclotron facility at the $\mathrm{Na}$ tional Superconducting Cyclotron Laboratory.

While the parameters of the simulation could be adjusted to match experimental data for plastic scintillators $[3,4]$, the question of how well the simulation described a converter-scintillator combination in the energy region of interest remained open. Now we are able to compare the predictions of our simulation with the measured data. For this purpose, the experimental setup was closely modeled in GEANT (see Fig. 5). The simulated setup was however exactly symmetrical, so that no correction had to be applied. The Monte Carlo code generated neutrons with random energies in the range of interest, emitted at random angles into a forward cone containing the detector setup. Veto detectors were included in the simulation. Charged particles were created according to the cross-section tables of GEANT where the neutrons passed through material. The light output generated by the charged particles that were tracked through the scintillator was accumulated for each detector block and had to surpass the software threshold in order for the neutron to be counted as detected. In addition, the light output was attenuated according to the distance to either end of the scintillator block, and the signal on both ends of one scintillator block had to surpass the hardware

\footnotetext{
${ }^{1}$ The hadron package FLUKA is employed except for neutrons below $20 \mathrm{MeV}$, where MICAP takes over.
} 


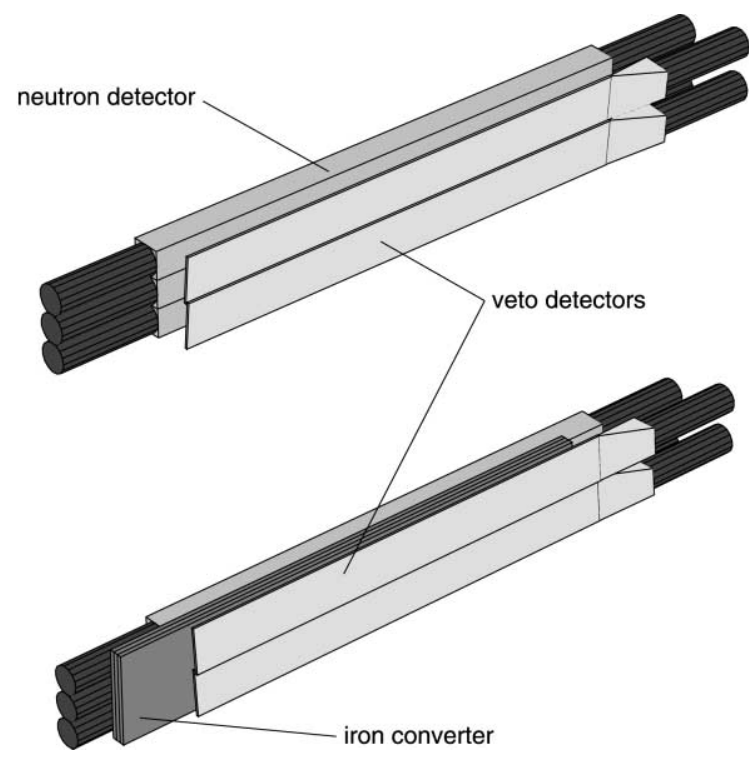

Fig. 5. View of the detector setup used for the GEANT simulation.
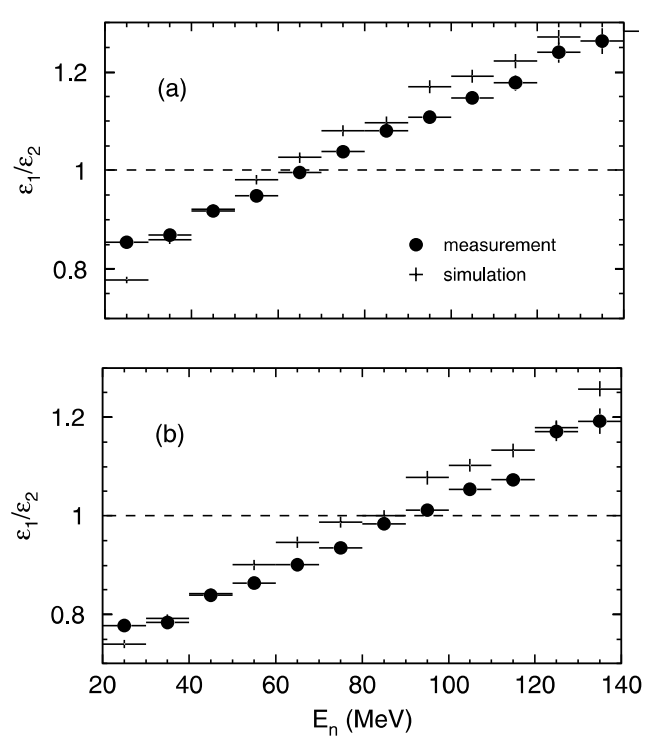

Fig. 6. Comparison of experimentally determined efficiency ratios (filled circles) and data from GEANT (FLUKA/MICAP) simulations (cross-hairs) employing a $3 \mathrm{~cm}$ iron converter. For the data in the upper panel (a), a 2 MeVee threshold cut was applied, the lower panel (b) shows the results for a $5 \mathrm{MeVee}$ cut.

threshold as well, otherwise the event would not be counted. A position-cut ensured that only the area of the detector that was properly covered by veto detectors and the iron converter was used. In the end, the number of neutrons detected in the converter-equipped detector set was compared to the number of detected neutrons from the detector set without converter, just as in the experimental analysis. The number of neutrons per energy bin only determines the statistical error for the efficiency ratio corresponding to that bin. Therefore the energy distribution of neutrons has no effect on the efficiency-ratio curves.

Results of the simulation for the $3 \mathrm{~cm}$ converter, both with a 2 and $5 \mathrm{MeVee}$ threshold cut, are displayed in Fig. 6 in comparison with the experimental data. The overall agreement is good, although the simulation slightly over-predicts the effectiveness of the converter at energies above $60 \mathrm{MeV}$.

\section{Conclusion}

This measurement corroborates our results from simulations in that passive iron converters can already be effectively used to enhance the neutron detection efficiency at energies below 200 $\mathrm{MeV}$. We found experimental evidence for a 10 $20 \%$ increase in detection efficiency at neutron energies between 100 and $140 \mathrm{MeV}$ for a combination of a 2 or $3 \mathrm{~cm}$ iron converter with $6 \mathrm{~cm}$ of plastic scintillator. Furthermore, our GEANT (FLUKA/MICAP) simulations reproduce the experimental results.

This measurement yields valuable information for the design of the large-area high-efficiency modular neutron array (MoNA) at the NSCL. This detector will make use of the neutron-rich rare-isotope beams of the coupled cyclotron facility, and is optimized for the $100-200 \mathrm{MeV}$ energy range. As the measurement presented here shows, the use of passive converters can significantly enhance detection efficiency at these energies.

\section{Acknowledgements}

This work was supported by the US National Science Foundation under grant PHY 95-28844. 


\section{References}

[1] Th. Blaich et al., Nucl. Instr. and Meth. A 314 (1992) 136.

[2] T. Nakamura et al., Phys. Lett. B 331 (1994) 296.

[3] R.A. Cecil, B.D. Anderson, R. Madey, Nucl. Instr. and Meth. 161 (1979) 439.

[4] R.L. Craun, D.L. Smith, Nucl. Instr. and Meth. 80 (1970) 239.

[5] Detector Description and Simulation Tool GEANT, Version 3.21, CERN Program Library, Geneva.
[6] GEANT-Detector Description and Simulation Tool, CERN, Geneva 1993, edition March 1995 (cited Nov. 2001); Documentation available at: http://wwwinfo.cern.ch/ asd/geant/.

[7] P.A. Aarnio et al., Fluka user's guide, Technical Report TIS-RP-190, CERN, 1987, 1990.

[8] A. Fassò, A. Ferrari, J. Ranft, P.R. Sala, G.R. Stevenson, J.M. Zazula, FLUKA92, in: Proceedings of the Workshop on Simulating Accelerator Radiation Environments, Santa Fe, USA, 11-15 January 1993. 